\title{
Irrational prescribing: myths and facts
}

According to $\mathrm{WHO}$, irrational prescribing is a disease which is difficult to treat but prevention is possible. ${ }^{1}$ There are many factors which contributes for this irrational prescribing like patient factors, practitioners/doctor factors, influence from pharmaceutical industry or combination of all.

New medical undergraduates should be well trained regarding rational prescribing, proper diagnosis and adequate dosing, frequency etc in their course as per MCI Pharmacology curriculum. So when they start their own clinic, he/she prescribes only an antihistamine for the patient with common cold for two days at bed time and asks the patient to come after two days but on other side the mind-set of the patient will be such that there exists a pill for every illness which makes them to think that particular doctor has not prescribed more drugs which may be due to lack of knowledge and so immediately the patient will go to another doctor who will prescribe at least 3 drugs one antihistamine, one antibiotic and analgesic for the common cold because of which the mind-set of people gets corrupted. Thus, the undue pressure by the patients on the health care providers/practioners to prescribe a greater number of drugs is one of the most troublesome factors which contributes for irrational use of medicines. Macfarlane et al had also proved that in the treatment of acute lower respiratory tract infections the inclusion of antibiotic in the prescription was around $74 \%$, of which patient pressure was the main reason in more than half. ${ }^{2}$

Second important factor is prescribing the drugs in generic name. Medical council of India is insisting the practitioners to prescribe drugs only in generic name but there are no stringent rules among the pharmacists to sell the drugs under generic name. Finally, the major role is played by the pharmaceutical industry by providing promotional incentives like gifts/travel arrangements to conferences which will also add up to irrational prescribing of medicines.

Another most unrevealed fact is that if a medical shop is close to the doctor's clinic who will prescribe generic drugs and will ask the patient to get those generic drugs in specific outlet and not in nearby medical shop. However, the patients do not abide the doctor's instructions. Often the patients go directly to the pharmacy to avoid doctor fee, wherein the pharmacists who are not qualified personnel. ${ }^{3}$ prescribe drugs without doctor's prescription. This voluntary behaviour of the patients to the pharmacists is often misused by the pharmacists to spread false conceptions about the doctor and his popularity as the doctor is not sending his patients to this medical shop but to generic outlet. Moreover, the pharmacist will sell the drugs from the company which gives highest incentive for him though the drugs with same efficacy are available at lowest cost which again contribute to irrational use of medicines.

Though it has been mentioned in some textbooks that doctors prescribe unnecessary injections, there lies a hidden truth that most of the patients request the doctors to prescribe injection for at least placebo effect. Mao et al had also proved that overuse of injections was more in rural areas when compared to urban in a study conducted in china. ${ }^{4}$ Some other reasons which influences the irrational use of drug were lack of diagnostic facilities mainly in rural areas which promotes the practitioners to prescribe more drugs (polypharmacy) and also because of improper communication between practitioners and the patient due to lack of time.

So, the questions put forth here are: 1 . Who will educate the patients? 2. Who will control the pharmacists on prescribing drugs without doctor's prescription? 3. Who will take the responsibility for the quality of drug provided? 4. Who will control pharmaceutical industries for giving incentives? In spite of all the legal policies and authority available to prevent this dreadful disease that endangers life of every human, irrational prescribing still remains a disease for which treatment is difficult but can be preventable but who, how and when it remains unknown.

Nandhini Saravanabavan, Sudar Codi R., Manimekalai K.*

Department of Pharmacology, Mahatma Gandhi Medical College and Research Institute, Puducherry, India

*Correspondence to Dr. Manimekalai K., E-mail: manimekalaik@mgmcri.ac.in

\section{REFERENCES}

1. World Health Organization. Teacher's Guide to Good Prescribing. World Health Organization; Geneva, Switzerland. 2001.

2. Macfarlane J, Holmes W, Macfarlane R, Britten N. Influence of patient's expectations on antibiotic 
management of acute lower respiratory tract illness in general practice: Questionnaire study. BMJ. 1997;315:1211-4.

3. Chandy SJ. Consequences of irrational use of antibiotics. Indian J Med Ethics. 2008;5(4):174-5.

4. Mao W, Vu H, Xie Z, Chen W, Tang S. Systematic review on irrational use of medicines in China and Vietnam. Plos one. 2015;10(3):1-18.
Cite this article as: Saravanabavan N, Codi SR, Manimekalai K. Irrational prescribing: myths and facts. Int J Basic Clin Pharmacol 2021;10:612-3. 